\title{
Slow and Stopped Light in Metamaterials
}

\author{
(Invited Paper) \\ Ortwin Hess and Kosmas Tsakmakidis \\ Advanced Technology Institute and Department of Physics, Faculty of Engineering and Physical Sciences, \\ University of Surrey, Guildford, Surrey, GU2 7XH United Kingdom
}

\begin{abstract}
We demonstrate the deceleration of guided electromagnetic waves propagating along an adiabatically tapered negative-refractive-index metamaterial heterostructure and show that light can ideally be brought to a complete halt. It is analytically shown that, in principle, this method simultaneously allows for broad bandwidth operation (since it does not rely on group index resonances), large delay-bandwidth products (since a wave packet can be completely stopped and buffered indefinitely) and high, almost $100 \%$ in/out-coupling efficiencies. The halting of a monochromatic field component travelling along the heterostructure is demonstrated on the basis of a wave analysis and confirmed in a pertinent ray analysis, which unmistakably illustrates the trapping of the associated lightray and the formation of a double light-ray cone (optical clepsydra) at the point where the ray is trapped. This method for trapping photons conceivably opens the way to a multitude of hybrid optoelectronic devices to be used in quantum information processing, communication networks and signal processors and may herald a new realm of combined metamaterials and slow light research
\end{abstract}

\section{INTRODUCTION}

An ability to controllably and all-optically delay or stop optical signals may have enormous implications for optical telecom networks and signal processors, and has recently excited the engineering and scientific community [1]. Specific applications of "slow-light" include random access memories, data synchronization, pattern correla-tion, optical delay lines, enhanced light-matter interactions and network buffering. A key feature of telecom-munication systems with a global net of optical fibres is the use of routers to switch the transmitted light-pulses from one fibre to another, so that they end up at the desired destination. Unfortunately, at present, buffering is very slow and creates severe data traffic congestion, and is generally believed to be responsible for slowing-down the speed of optical networks by a factor of 1000 [2]. Seemingly paradoxically, making light travel slower rather than faster at such cross-points is the best way to meet the networks high-speed challenges, which will become even greater in the foreseeable future as the transmission capacity of optical fibres increases.

Until now, the prime difficulty in designing an all-optical network router was finding a way of temporarily storing and buffering the packets of information. Optical engineers have generally asserted that optical data cannot be stored statically and must be processed and switched on the fly [2]. The basic reason for this con-clusion was that buffering an optical signal by dramatically reducing the speed of light itself, was thought not to be possible. Recently, however, major scientific breakthroughs within the optical engineering community helped to overturn this notion and proved that it is possible to bring light to a complete standstill. Indeed, a variety of methods have been proposed as a means of producing slow or stopped light, including electromagnetically induced transparency (EIT) [3], quantum-dot semiconductor optical amplifiers (QD-SOAs) [4], photonic crystals (PCs) [5], coherent population oscillations (CPOs) [6], stimulated Brillouin scattering (SBS) [7] and surface plasmon polaritons (SPPs) in metallodielectric waveguides [8], [9]. Most of these methods, though interesting, bear inherent (i.e. inescapable) weaknesses that may hinder their practical applications within the presently described context. So far EIT uses ultracold or hot gases and not solid-state materials, while CPO and SBS are very narrowband (typically, several $\mathrm{MHz}$ ) owing to the narrow transparency window of the former and the narrow Brillouin gain bandwidth of the latter. This, combined with the strong impedance mismatch in the slow-light regime, makes launching of the incoming light to a slow mode very difficult.

In an effort to overcome these limitations, a fundamentally new approach has recently been proposed [10]. Based on the presence of negative Goos-Hänchen phase-shifts at the interfaces between positive- and negative-index materials, due to the power-flow direction inside the negative-index regions being opposite to that in the positive-index regions (see Fig. 1), a pronounced deceleration of the guided electromagnetic waves was observed in simulation. We note that precisely the same principle of a negative phase shift at dielectric/metal inter-faces, which allows for broadband deceleration of light (Trapped Rainbow).

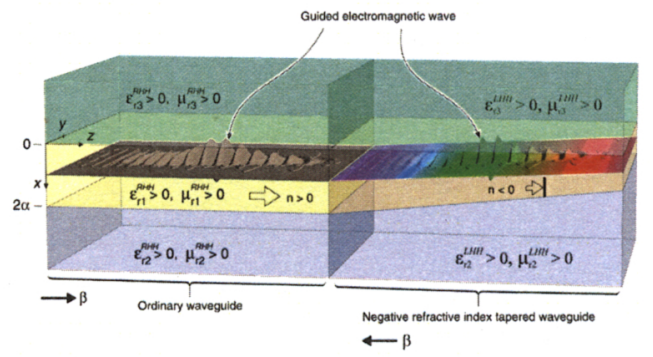

Fig. 1. Schematic illustration of an oscillatory wave guided along an ordinary dielectric waveguide and coupled to an adiabatically tapered lefthanded heterostructure. Each frequency component (colour) of the wave stops at correspondingly different point inside the tapered negative-index waveguide, forming a trapped rainbow. 


\section{NUSOD 2008}

\section{Slow Light in Metamaterial Heterostructures}

In this contribution we discuss how efficient slow and stopped light can be realized inside axially varying, adiabatically tapered, negative refractive index (NRI) metamaterial heterostructures. At the heart of the scheme is realization of a negative refractive index through metamaterials - materials exhibiting simultaneously negative electric permittivity and magnetic permeability [13], [14], [15]. This method for producing slow-light turns out to be remarkably simple and bears a number of serious advantages compared to previously proposed ways of decelerating optical signals. The heterostructures investigated here can be designed for monomode operation in the desired frequency range, while the control of the group velocity can be achieved by appropriate tuning of the microphotonic structure, e.g. through gain to (via the Kramers-Kronig relationship) locally modify the refractive index, or via optically-induced attractive/repulsive forces exerted on the heterostructures to manipulate the thickness of its core. Figure 2 shows a snapshot from the propagation and complete halting of a monochromatic wave of frequency $f=1 \mathrm{THz}$, carrying a total, conserved, time-averaged powerflow $82.6 \mu \mathrm{W} / \mathrm{m}^{2} / \mathrm{sec}$. The core layer has refractive index $n_{1}=$ ?5, while the refractive indices of the two dielectric cladding layers are $n_{2}=1.6$ and $n_{3}=1.5$. The wave propagates smoothly down the waveguide, and at the critical thickness of $a k_{0}=0.55$ it altogether stops $\left(v_{g}=0\right)$. We also note that, while propagating, the wave is in the "negative phase velocity" mode, i.e. the directions of the time-averaged power flow and the longitudinal propagation constant $\beta$ are antiparallel. It is interesting to note that, owing to the anomalous frequency dispersion associated with the metamaterial heterostructure, larger wave frequencies (i.e. smaller free-space wavelengths) are stopped at larger core thicknesses, as illustrated in Fig. 1.

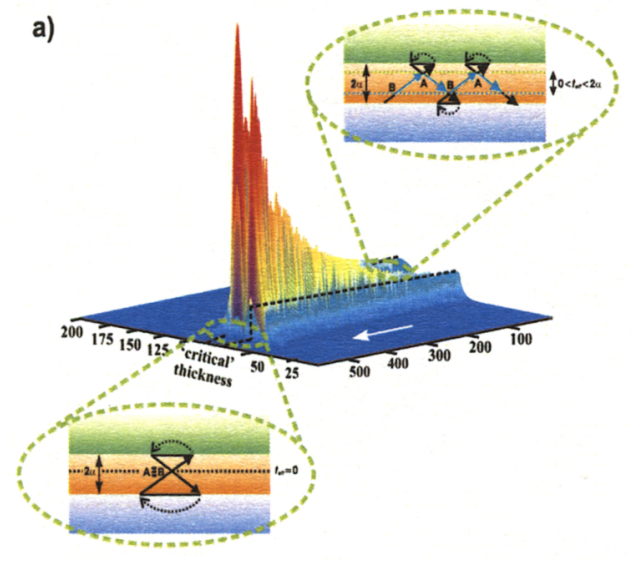

Fig. 2. Association of wave propagation inside the adiabatically tapered negative refractive index heterostructure with the corresponding zigzag ray analysis for different guide widths .

An intuitive picture of the physics associated with the slowing down and stopping of the wave can be obtained by tracing the trajectory of the associated light ray (of one of the monochromatic waves pertaining to a wave-packet) along its zigzag path. To this end, let us assume that a ray of p-polarised light impinges upon one of the interfaces between the negativeindex and the (normal) positive index layer with angle $\theta$. In stark contrast to conventional dielectric waveguides, in the NRI heterostructures the effective thickness $t_{\text {eff }}$ of the centre guiding layer is always smaller (see Fig.2, top right inset) than the core physical thickness $2 \alpha$, and can become zero or even negative. An example of zero effective NRI waveguide thickness is illustrated in the bottom left inset of Fig. 2. For the set of optogeometric parameters corresponding to that inset, the two Goos-Hänchen phase shifts experienced by the ray upon hitting the two media interfaces are such that the light ray becomes permanently trapped, forming a double light-ray cone, which (in view of its characteristic hourglass form) we call optical clepsydra.

\section{CONCLUSION}

We have shown how guided electromagnetic energy can efficiently be brought slowed down whilst travelling inside axially varying NRI waveguiding heterostructures. By nature, the scheme invokes solid-state materials and, as such, is not subject to low-temperature or atomic coherence limitations. Moreover, it inherently allows for high in-coupling efficiencies and broadband function, since the deceleration of light does not rely on refractive index resonances. This method for trapping photons opens the way to a multitude of hybrid, optoelectronic devices to be used in quantum information processing, communication networks and signal processors, and conceivably heralds a new realm of combined metamaterials and slow light research.

\section{ACKNOWLEDGMENT}

The authors would like to thank the EPSRC for financial support.

\section{REFERENCES}

[1] L. V. Hau, S. E. Harris, Z. Dutton, and C. H. Bhroozi, Nature 397, 594 (1999).

[2] D. J. Blumenthal, et al., Proc. IEEE 82, 1650 (1994).

[3] C. Liu, Z. Dutton, C. H. Behroozi, and L. V. Hau Nature (London) 409, 490 (2001).

[4] E. Gehrig, M. van der Poel, J. Mrk, and O. Hess, IEEE J. Quantum Electron. 42, 1047 (2006).

[5] Y. A. Vlasov, M. O'Boyle, H. F. Hamann, and S. J. McNab, Nature (London) 438, 65 (2005).

[6] M. S. Bigelow, N. N. Lepeshkin, and R. W. Boyd, Science 301, 200 (2003).

[7] Y. Okawachi, et al., Phys. Rev, Lett. 94, 153902 (2005).

[8] M. I. Stockman, Phys. Rev. Lett. 93, 137404 (2004).

[9] A. Karalis, E. Lidorikis, M. Ibanescu, J. D. Joannopoulos, and M. Solja, Phys. Rev. Lett. 95, 063901 (2005).

[10] K. L. Tsakmakidis, A. D. Boardman, and O. Hess, Nature (London) 450, 397 (2007).

[11] G. V. Eleftheriades and K. G. Balmain, Negative Refraction Metamaterials: Fundamental Principles and Applications (Wiley-IEEE, New Jersey, 2005).

[12] A. Al and N. Engheta, Phys. Rev. B 75, 024304 (2007).

[13] T. J. Yen, W. J. Padilla, N. Fang, D. C. Vier, D. R. Smith, J. B. Pendry, D. N. Basov, and X. Zhang, Science 303, 1494 (2004).

[14] A. N. Grigorenko, A. K. Geim, H. F. Gleeson, Y. Zhang, A. A. Firsov, I. Y. Khrushchev, and J. Petrovic, Nature (London) 438, 17 (2005)

[15] V. M. Shalaev, Nature Photonics 1, 41 (2007). 